\title{
$\Delta a$ and Strömgren photometry of stars in the Renson-catalogue of Ap and Am stars ${ }^{\star}$
}

\author{
H.M. Maitzen, R. Pressberger, and E. Paunzen \\ Institut für Astronomie der Universität Wien, Türkenschanzstr. 17, A-1180 Wien, Austria \\ e-mail: surname@astro.ast.univie.ac.at
}

Received July 17; accepted September 3, 1997

\begin{abstract}
We have observed 131 stars of Renson's (1991) catalogue of Ap and Am stars both in the Strömgren \& Maitzen's (1976) $\Delta a$ system as a contribution to the photometric studies of the $\lambda 5200$ broad band flux depression feature in chemically peculiar stars. With few exceptions the probability grouping of Renson for membership in the CP2 group of peculiar stars is nicely reflected by peculiar values of $\Delta a$. Comparison with already available $\Delta a$ values yields slightly larger values due to a minor shift in the filter $g_{1}$ sampling the depression. As found by Maitzen \& Vogt (1983) the Geneva system peculiarity parameters correlate well with $\Delta a$. This study demonstrates the advantageous performance of a photoelectric photometer with a rapidly rotating filter wheel moving in a stop and go mode.
\end{abstract}

Key words: stars; chemically peculiar — stars; early type

\section{Introduction}

Maitzen (1976) introduced a 3 filter system with intermediate band width for measuring the depth of the broad flux depression around $5200 \AA$, which was shown to occur among the magnetic peculiar stars. In the division proposed by Preston (1974) these objects constitute the whole CP2 and part of the CP4 group (Helium weak stars). Maitzen \& Vogt (1983) have substantiated the original finding and definitely established with a very large sample of stars that the photometric index $\Delta a$ is a very efficient means for identifying magnetic CP-stars, enabling statistical studies in a non-subjective way and to much larger distances than the traditional spectroscopic detection method would allow.

Send offprint requests to: H.M. Maitzen

* Tables 3 and 4 are also available in electronic form at the CDS via anonymous ftp to cdsarc.u-strasbg.fr (130.79.128.5) or via http://cdsweb.u-strasbg.fr/Abstract.html
The non-magnetic branch of CP-stars comprising CP1 (Am stars) and CP3 (Hg Mn stars) did not show individually peculiar values of $\Delta a$ albeit as groups they deviated from normal stars in the $1-2 \sigma$ range. This value was based on rather low number statistics and called for better foundation. This is one of the goals of the present investigation.

A second aim of this work is to contribute to the Renson (1991) catalogue of Ap and Am stars (6684 objects) which lists also Ap-detections obtained by the $\Delta a$ system. In cases where spectroscopic evidence had been published, our $\Delta a$ values can give additional hints for the assessment of a star to be peculiar in view of the probability for peculiarity as given by Renson. This probability was indicated by him using four different symbols:

$\begin{array}{ll}* & \text { well established } \\ \text { blank } & \text { very probable } \\ ? & \text { less probable } \\ \backslash & \text { erroneous. }\end{array}$

In this connection it should be mentioned that already the very first "catalogue" of peculiar A stars - a list of 9 stars - brought to the public by Maury (1897) hundred years ago contains one erroneous classification as Silicon star (81 UMa) which has not been confirmed by subsequent publications.

While the number of very probably erroneous peculiarity classifications in the Renson catalogue is of the order of a few percent, the number of objects with a question mark comprises more than 30 percent and further evidence on the nature of these objects, e.g. by our photometry seems to be desirable.

This is also valid for those stars in the Renson catalogue which do not exhibit a proper CP1-4 type, but just a HD classification without direct indication of the reason why they were included in the catalog.

A further goal will be the comparison with the list of $\Delta a$ values published by Lebedev (1986), in which also spectrophotometric data were used to synthesize $\Delta a$. 
Helpful and clarifying will be the comparison with the peculiarity parameters of the Geneva system derived from the catalogue of Rufener (1988).

Since about 20 percent of our programme stars lack photometry in the Strömgren system (which we need for deriving $\Delta a$ values) we have included such measurements. As a consequence, we have measured both standard and programme stars in 6 filters: $u v b y g_{1} g_{2}$, using a novel photometer with a rapidly rotating filter wheel in stop and go motion. This instrumentation has been devised to enable photometric measurements also under non-excellent weather conditions.

Table 1. Used filter system

\begin{tabular}{ccccl}
\hline Filter & $\lambda_{\mathrm{C}}[\mathrm{nm}]$ & $\mathrm{HW}[\mathrm{nm}]$ & $T_{\max }[\%]$ & \multicolumn{1}{c}{ type } \\
\hline$u$ & 352.6 & 16.0 & 42 & Schott UV-DAD \\
$v$ & 410.1 & 21.4 & 43 & Schott DMZ \\
$b$ & 467.0 & 19.5 & 63 & Schott DMZ \\
$g_{1}$ & 501.7 & 11.0 & 82 & Anders, Germany \\
$g_{2}$ & 521.2 & 12.0 & 82 & Anders, Germany \\
$y$ & 547.3 & 18.8 & 58 & Schott DMZ \\
\hline
\end{tabular}

\section{Observations and reduction}

The measurements were carried out at the $1 \mathrm{~m}$ telescope of the Purgathofer Observatory (Klosterneuburg, N/W of Vienna) by one of us (RP). The photometer includes an EMI 6256B photomultiplier Peltier cooled and operated at $1170 \mathrm{~V}$, and a rapidly rotating filter wheel which nevertheless stops for 60 milliseconds at each filter and moves during 20 milliseconds to the next one. Thus, one turn of the filter wheel takes nearly half a second (480 milliseconds). Since the individual integrations of usually 200 turns of the wheel were coadded in each filter, the results should be practically simultaneous.

The filter set is presented in Table 1. A detailed description of the photometer has been given by Pressberger \& Stoll (1993). Because of the bright sky of nearby Vienna a rather narrow diaphragm of $7^{\prime \prime}$ was used. Therefore, special care was taken to recenter the star under observation at the beginning of each series (lasting about 100 seconds). The programme stars were observed only on one night each, usually by two sets of filter measurements, but sometimes also 3 and very rarely 4 sets. The dead time correction was 65 nanoseconds.

In what follows we will present all photometric values in the Strömgren and $\Delta a$ systems in units of 0.001 mags.

Table 2 contains the list of observing nights with average external accuracies of the Strömgren parameters and $\Delta a$ (for non-peculiar stars) together with the zero points of the normality line. The external errors were derived from the differences of observed and published Strömgren indices. It is very instructive to notice that the average nightly scatter of $\Delta a$ values for non-CP2 stars (= practically normal stars where the small number of deviating cases among the other groups discussed below was disregarded) on 9 nights is smaller than 4 mmags and only on 4 nights between 4 and 5 mmags. This accuracy is nearly identical with that obtained by Maitzen (1976) in his introductory paper on $\Delta a$ photometry where he could rely on the excellence of the Chilean Atacama dessert sky (albeit in traditional photometric observing mode). The obviously larger average errors in the Strömgren parameters are due both to their definition (implying the influence of atmospheric extinction not present in $\Delta a$ ) and to the error level contained in the published values. In some cases intrinsic variability may also have contributed to this aspect. Table 3 lists the differences of the published Strömgren indices and our own values. Figure 1 shows the histogram for the $\Delta(b-y)$ values for which we obtain a standard deviation of 6.8 mmags from a Gaussian fit for 110 stars. Since according to Table 2 different average night deviations have been obtained, it is not surprising that the histogram is not perfectly approximated by a Gaussian distribution. For the $\Delta m_{1}$ values we calculate $7.1 \mathrm{mmags}$ as standard deviation for 109 stars (we discard the deviating value for HD 209711 discussed below). For the case of the $c_{1}$ parameter we derive 9.5 mmags (again for 110 stars). The $\Delta a$ values yield a much better Gaussian fit with corresponding standard deviation of only 3.1 mmags.

Table 4 exhibits the results of our Strömgren and $\Delta a$ photometry together with their mean errors (i.e. internal precision based on the individual observations during the relevant night).

The $\Delta a$ values were obtained as usual by forming the difference between the observed $a$-value and the $a_{0}$-value of the normality line at the same $b-y$ as the concerning star. The normality line, i.e. the locus of non-peculiar stars in the $a$ vs. $b-y$ diagram, could be represented by a straight line with essentially constant slope but slightly varying zero point $a_{i}$ (see Table 2 ).

The individual normality lines can thus be represented by:

$a_{0}=a_{i}+0.115(b-y)$

The parameters of those normality lines were fixed by measurements of a sample of 12 non-peculiar stars. As explained below, the majority of $\mathrm{Am}, \mathrm{HgMn}$ and $\mathrm{He}$ abnormal stars line up very closely to the normality lines and thus reinforce the determination of the null-line of peculiarity.

Our sample of peculiar stars can be characterized by the following criteria:

- From mechanical reasons the upper declination limit was set to $70^{\circ}$. From reasons concerning airmass and the southern sky illumination by the city of Vienna we set a lower limit to declination at $0^{\circ}$. 
Table 2. Observing log with the estimated errors for all nights

\begin{tabular}{ccrrrrr}
\hline night & yymmdd & $a_{i}$ & $\sigma(b-y)$ & $\sigma\left(m_{1}\right)$ & $\sigma\left(c_{1}\right)$ & $\Delta a$ \\
\hline 1 & 930320 & 0 & 10.5 & 7.4 & 7.7 & 3.7 \\
2 & 930416 & 0 & 6.8 & 7.0 & 4.6 & 3.1 \\
3 & 930420 & 2 & 7.3 & 8.9 & 8.3 & 4.9 \\
4 & 940403 & 5 & 9.7 & 6.3 & 12.3 & 4.1 \\
5 & 940407 & 11 & 8.4 & 5.8 & 13.3 & 2.3 \\
6 & 940420 & 11 & 5.2 & 9.3 & 9.3 & 3.7 \\
7 & 940503 & 11 & 5.5 & 8.3 & 9.0 & 3.5 \\
8 & 940513 & 9 & 16.5 & 10.2 & 14.4 & 5.0 \\
9 & 940601 & 5 & 5.0 & 13.9 & 19.4 & 4.4 \\
10 & 940929 & 12 & 10.3 & 14.2 & 11.3 & 2.3 \\
11 & 941009 & 12 & 4.5 & 8.1 & 7.7 & 3.7 \\
12 & 941010 & 12 & 6.5 & 10.0 & 18.6 & 3.3 \\
13 & 941025 & 5 & 5.1 & 11.9 & 20.8 & 2.6 \\
14 & 941201 & 8 & 6.2 & 11.0 & 32.3 & 4.6 \\
\hline & & & & & &
\end{tabular}

- The brightness limits were 4 and 8 mags.

- The 131 objects of our programme are distributed in R.A. from $7 \mathrm{~h} 42 \mathrm{~m}$ to $13 \mathrm{~h} 40 \mathrm{~m}$ (82 stars) and from $19 \mathrm{~h} 04 \mathrm{~m}$ until $22 \mathrm{~h} 27 \mathrm{~m}$ (49 stars). All types of objects from the Renson catalogue were regarded as potential objects of this programme, and the actual observing conditions were the main reason for the definite choice of objects in the R.A. interval mentioned.

Reddening corrections in $b-y$ had to be applied only to very few stars of our sample in order to obtain intrinsic $\Delta a$ values. We derived the following intrinsic $(b-y)_{0}$ values: for the Silicon stars HD 179527 and HD 204815 -37 and -24 , resp.,

for the $\mathrm{Hg}$ Mn star HD $178065-42$,

for the He wk star HD $212454-70$ and

for the He str star HD $209339-117$.

Table 3. Comparison of our photometry with the literature

\begin{tabular}{rcrrr}
\hline RNS & HD & $\Delta b-y$ & $\Delta m_{1}$ & $\Delta c_{1}$ \\
\hline 17470 & 63312 & -16 & -2 & 6 \\
18200 & 66068 & -8 & 4 & -6 \\
18950 & 68457 & -2 & -7 & -3 \\
18990 & 68542 & -4 & 8 & 24 \\
19050 & 68703 & -3 & 13 & -6 \\
19060 & 68725 & -16 & -1 & 4 \\
19260 & 69682 & -8 & 8 & 0 \\
19550 & 70826 & 13 & 0 & -7 \\
19750 & 71534 & 4 & -9 & -15 \\
19850 & 71866 & 10 & -20 & -15 \\
19950 & 72208 & 6 & 0 & -3 \\
\hline
\end{tabular}

Note to Table 3.:

The full content of this Table is available from one of the authors (HMM) or via Simbad.

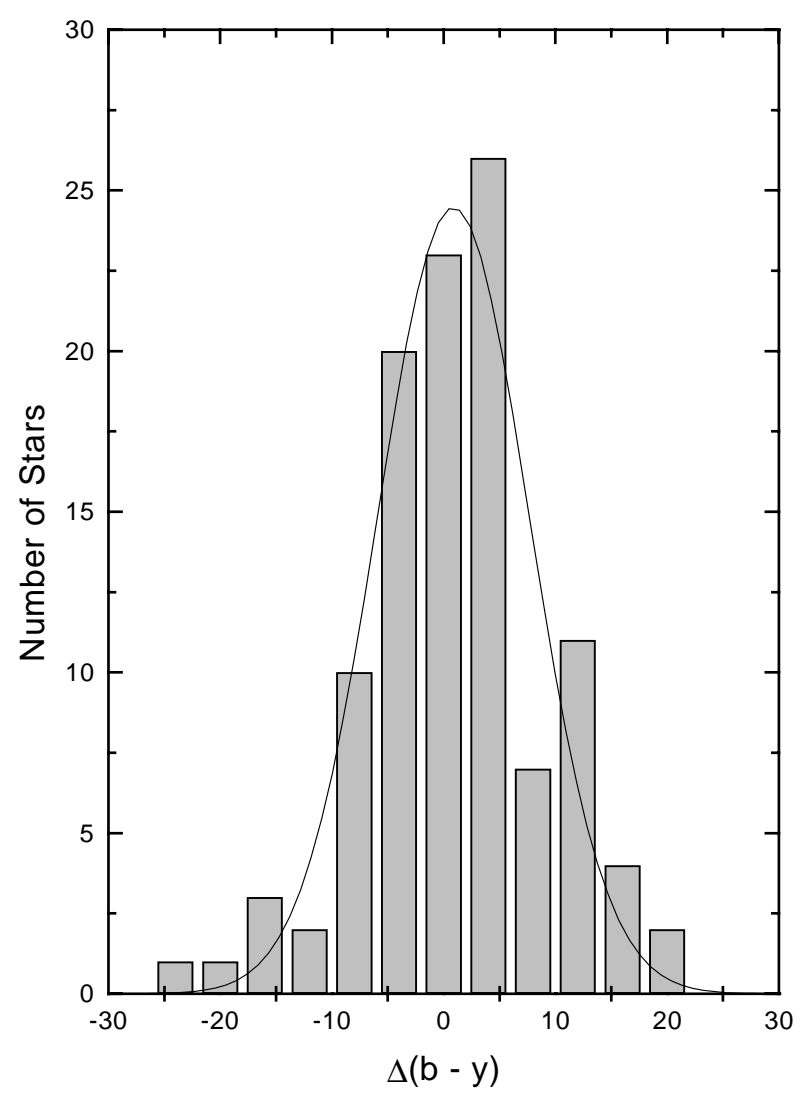

Fig. 1. Histogram for all $\Delta(b-y)$ values from Table 3

\section{Results and discussion}

\subsection{CP2-stars}

For this group and the other peculiarity groups we decided to present the results graphically in such a way that the original $a$-values rather than the $\Delta a$ values were used. Since the slope of the normality line is a constant as described above we had only to choose a zero point for the $a$-values, which we set to 0 for $b-y=0$.

Figure 2 exhibits the diagram $a$ vs. $b-y$ for the 24 stars of our sample which according to Renson (1991) are CP2 stars, thus magnetic peculiar stars of the upper main sequence. In order to show the effect of Renson's grouping in 4 probability classes we have chosen different symbols for them. We notice that all stars with at least 3 standard deviations above the normality line are in his categories "well established" or "very probable". Only one star with question mark is obviously significantly above the line: HD 204411 with $\Delta a=19$, very similar to the result of Maitzen \& Seggewiss (1980) with $\Delta a=16$. The erroneous attribution of CP2 character for HD 118214 is reflected by its completely normal photometric behaviour $(\Delta a=3)$.

Comparison with Maitzen (1976) is possible for three cases. In the sense this work minus Maitzen (1976) the dif- 
Table 4. a. Programme stars

\begin{tabular}{|c|c|c|c|c|c|c|c|c|c|c|c|c|c|}
\hline$\overline{\mathrm{CP}}$ & night & RNS & HD & $\operatorname{mag}_{V}$ & $b-y$ & m.e. & $m_{1}$ & m.e. & $c_{1}$ & m.e. & $\Delta a$ & m.e. & Spt. \\
\hline 1 & 1 & $15290 ?$ & 56152 & 7.0 & 25 & 4 & 189 & 6 & 1063 & 7 & -1 & 3 & A1-A2 \\
\hline 1 & 1 & 17080 & 62257 & 7.6 & 78 & 2 & 222 & 1 & 1001 & 9 & 5 & 1 & A4-A9 \\
\hline 1 & 1 & $17470 ?$ & 63312 & 7.1 & 86 & 4 & 222 & 6 & 948 & 6 & 4 & 7 & A2- \\
\hline 1 & 4 & 18200 & 66068 & 7.0 & 202 & 1 & 228 & 3 & 686 & 4 & 5 & 1 & A2-F3 \\
\hline 1 & 4 & $18580^{*}$ & 67317 & 7.9 & 171 & 9 & 247 & 6 & 710 & 2 & 6 & 4 & A2-F2 \\
\hline 1 & 1 & $18950 ?$ & 68457 & 6.5 & 123 & 1 & 180 & 4 & 1026 & 3 & 0 & 1 & A7- \\
\hline 1 & 4 & 18990 & 68542 & 7.6 & 268 & 6 & 265 & 3 & 645 & 5 & -2 & 4 & F3-F7 Sr \\
\hline 1 & 1 & $19050 ?$ & 68703 & 6.4 & 177 & 2 & 208 & 4 & 819 & 2 & 4 & 2 & A8- DD \\
\hline 1 & 4 & 19060 & 68725 & 6.7 & 252 & 7 & 204 & 3 & 706 & 13 & -1 & 3 & F2-F6 \\
\hline 1 & 1 & $19260 ?$ & 69682 & 6.5 & 185 & 4 & 194 & 5 & 707 & 4 & -6 & 5 & F0- DD \\
\hline 1 & 4 & 19550 & 70826 & 73 & 390 & 0 & 245 & 2 & 634 & 13 & -7 & 2 & A3-F8 \\
\hline 1 & 4 & 19750 & 71534 & 7.0 & 397 & 5 & 260 & 5 & 577 & 2 & 2 & 3 & F2-F9 \\
\hline 2 & 4 & $19850^{*}$ & 71866 & 6.8 & 4 & 2 & 284 & 5 & 838 & 10 & 60 & 4 & A1 EuSrSi \\
\hline 3 & 4 & $19950 ?$ & 72208 & 6.8 & -18 & 2 & 131 & 5 & 893 & 8 & 2 & 9 & B9 MnHg \\
\hline 1 & 4 & 19970 & 72280 & 7.6 & -1 & 4 & 185 & 2 & 1043 & 12 & 12 & 6 & A9-F1 \\
\hline 1 & 4 & 20170 & 72792 & 7.6 & 216 & 12 & 240 & 3 & 698 & 9 & 0 & 3 & F0 DD \\
\hline 1 & 1 & $20220 ?$ & 72942 & 7.5 & 68 & 2 & 204 & 3 & 1041 & 11 & -12 & 9 & A2- \\
\hline 1 & 1 & 20450 & 73618 & 7.3 & 93 & 3 & 234 & 2 & 970 & 14 & 1 & 3 & A3-F0 \\
\hline 1 & 1 & 20460 & 73619 & 7.5 & 133 & 4 & 238 & 2 & 829 & 13 & 0 & 7 & A3-F2 \\
\hline 1 & 5 & 20480 & 73666 & 6.6 & -8 & 1 & 174 & 0 & 1097 & 0 & 1 & 1 & A1- \\
\hline 1 & 5 & 21540 & 76461 & 6.9 & 158 & 3 & 245 & 3 & 753 & 5 & 4 & 1 & A5-F3 \\
\hline 3 & 5 & $21860^{*}$ & 77350 & 5.5 & -23 & 2 & 133 & 2 & 1012 & 2 & 1 & 1 & B9 SrCrHg \\
\hline 1 & 5 & 22010 & 77745 & 6.9 & 142 & 6 & 245 & 3 & 818 & 1 & 5 & 1 & A4-F3 \\
\hline 1 & 5 & $22160 *$ & 78209 & 4.5 & 160 & 3 & 236 & 1 & 770 & 0 & 0 & 1 & A2-F3 \\
\hline 3 & 5 & $22170^{*}$ & 78316 & 5.3 & -35 & 2 & 112 & 2 & 583 & 16 & 1 & 1 & B8 $\mathrm{MnHg}$ \\
\hline 1 & 5 & $22190^{*}$ & 78362 & 4.6 & 213 & 0 & 244 & 2 & 720 & 1 & 0 & 2 & A5-F6 \\
\hline 1 & 5 & $22420 ?$ & 79066 & 6.3 & 202 & 6 & 167 & 4 & 609 & 10 & -16 & 1 & A9- DD \\
\hline 4 & 5 & $22480^{*}$ & 79158 & 5.3 & -63 & 4 & 128 & 5 & 575 & 2 & 31 & 3 & B9 Hewk \\
\hline$?$ & 5 & $22540 ?$ & 79439 & 4.8 & 121 & 3 & 200 & 2 & 897 & 5 & -2 & 1 & A6 \\
\hline 4 & 6 & $22550 ?$ & 79469 & 3.9 & -35 & 2 & 139 & 1 & 969 & 5 & -2 & 0 & A0 Hewk \\
\hline 1 & 6 & 23320 & 82030 & 6.9 & 147 & 6 & 239 & 6 & 784 & 7 & -6 & 7 & $\mathrm{~A} 0-$ \\
\hline 1 & 6 & 23370 & 82191 & 6.6 & 42 & 3 & 191 & 1 & 989 & 6 & 3 & 1 & A1-F0 \\
\hline 1 & 6 & 23930 & 83886 & 6.5 & 50 & 2 & 206 & 2 & 1015 & 4 & 3 & 1 & A3- \\
\hline 1 & 6 & $24280 ?$ & 85040 & 6.1 & 152 & 9 & 210 & 4 & 923 & 1 & 3 & 4 & A7- \\
\hline$?$ & 6 & $24410 ?$ & 85504 & 6.0 & -22 & 0 & 134 & 4 & 1043 & 4 & 3 & 0 & $\mathrm{~A} 1$ \\
\hline 2 & 6 & $24690 ?$ & 86274 & 6.6 & -29 & 6 & 124 & 9 & 1029 & 3 & 6 & 2 & A0 Si \\
\hline 1 & 6 & 24750 & 86516 & 6.6 & 109 & 1 & 224 & 6 & 871 & 4 & -1 & 2 & A2- \\
\hline 1 & 6 & 24820 & 86902 & 7.2 & 166 & 5 & 236 & 16 & 741 & 18 & -3 & 7 & $\mathrm{~A} 0-$ \\
\hline 1 & 6 & $25190 ?$ & 88022 & 6.7 & 347 & 7 & 188 & 9 & 772 & 3 & -1 & 3 & $\mathrm{~A} 2-\mathrm{F}$ \\
\hline 3 & 7 & $25790 *$ & 89822 & 4.9 & -29 & 1 & 147 & 1 & 955 & 2 & 6 & 1 & A0 $\mathrm{HgSiSr}$ \\
\hline 2 & 7 & $25890^{*}$ & 90044 & 6.0 & -70 & 5 & 223 & 10 & 911 & 24 & 65 & 4 & B9 $\mathrm{SiCrSr}$ \\
\hline 2 & 7 & $26010^{*}$ & 90569 & 6.0 & -44 & 6 & 182 & 1 & 940 & 2 & 36 & 5 & A0 $\mathrm{SrCrSi}$ \\
\hline 1 & 7 & 26120 & 90931 & 6.9 & 197 & 1 & 229 & 1 & 704 & 2 & 6 & 1 & A8-F3 \\
\hline 2 & 7 & 26760 & 92728 & 5.8 & -26 & 1 & 149 & 3 & 1024 & 1 & 4 & 0 & A0 Si \\
\hline 1 & 7 & 26790 & 92769 & 5.5 & 104 & 0 & 189 & 2 & 897 & 2 & 1 & 2 & A2- \\
\hline 1 & 7 & $27160 ?$ & 93901 & 6.6 & 199 & 1 & 201 & 1 & 720 & 8 & 2 & 1 & A5- \\
\hline 2 & 7 & $27230 ?$ & 94334 & 4.7 & -12 & 2 & 148 & 1 & 1054 & 2 & 3 & 3 & $\mathrm{~A} 1 \mathrm{Si}$ \\
\hline 1 & 7 & $27280 ?$ & 94480 & 6.2 & 183 & 2 & 202 & 7 & 887 & 2 & 3 & 1 & A5-F3 \\
\hline 1 & 7 & 27440 & 95256 & 6.4 & 82 & 2 & 249 & 1 & 959 & 5 & 10 & 2 & A1- \\
\hline 1 & 3 & $27470 ?$ & 95310 & 5.1 & 140 & 1 & 188 & 1 & 1003 & 2 & 2 & 1 & A7- \\
\hline 1 & 8 & 27840 & 96528 & 6.5 & 119 & 3 & 203 & 9 & 901 & 4 & -2 & 0 & A3-A7 \\
\hline 1 & 8 & 28610 & 99302 & 7.3 & 143 & 9 & 191 & 5 & 804 & 14 & -9 & 2 & A3-F1 \\
\hline 1 & 8 & $28670^{*}$ & 99620 & 7.7 & 106 & 4 & 210 & 2 & 869 & 14 & 0 & 1 & A2-A9 \\
\hline 1 & 3 & 28740 & 99859 & 6.3 & 79 & 2 & 199 & 2 & 928 & 2 & -6 & 1 & A3-A9 \\
\hline 3 & 3 & 29210 & 101391 & 6.4 & -44 & 2 & 110 & 3 & 696 & 4 & 2 & 2 & B9 HgMn \\
\hline
\end{tabular}


Table 4. b. continued

\begin{tabular}{|c|c|c|c|c|c|c|c|c|c|c|c|c|c|}
\hline$\overline{\mathrm{CP}}$ & night & RNS & HD & $\operatorname{mag}_{V}$ & $b-y$ & m.e. & $m_{1}$ & m.e. & $c_{1}$ & m.e. & $\Delta a$ & m.e. & Spt. \\
\hline 1 & 8 & 29390 & 101968 & 7.9 & 21 & 3 & 189 & 1 & 1032 & 16 & 10 & 3 & $\mathrm{~A} 0-$ \\
\hline 1 & 8 & 29430 & 102056 & 7.0 & 29 & 1 & 162 & 4 & 1065 & 10 & -1 & 5 & $\mathrm{~A} 2-\mathrm{A} 7$ \\
\hline 1 & 3 & 29730 & 102942 & 6.2 & 192 & 3 & 234 & 4 & 728 & 4 & 6 & 1 & A3-F4 \\
\hline 2 & 8 & 29830 & 103498 & 7.0 & -9 & 3 & 185 & 2 & 1008 & 3 & 46 & 4 & $\mathrm{~A} 1 \mathrm{CrEuSr}$ \\
\hline 1 & 3 & $30150 ?$ & 104241 & 7.6 & 32 & 3 & 176 & 2 & 1050 & 5 & -4 & 3 & $\mathrm{~A} 1$ \\
\hline 1 & 2 & 30250 & 104513 & 5.2 & 158 & 2 & 192 & 2 & 751 & 3 & 1 & 2 & A4-A9 \\
\hline 1 & 8 & 30550 & 105601 & 7.4 & 165 & 10 & 205 & 17 & 776 & 5 & 4 & 6 & A2-F2 \\
\hline 1 & 2 & $30930 ?$ & 106887 & 5.7 & 87 & 1 & 222 & 1 & 929 & 5 & 7 & 3 & A3-F1 \\
\hline 1 & 8 & $31000 ?$ & 107131 & 6.5 & 99 & 5 & 189 & 3 & 951 & 1 & 2 & 2 & A3-A7 \\
\hline 1 & 8 & $31030^{*}$ & 107168 & 6.3 & 97 & 2 & 249 & 3 & 929 & 13 & 18 & 2 & A6-F1 \\
\hline 1 & 2 & $31080 ?$ & 107276 & 6.7 & 110 & 8 & 200 & 5 & 907 & 11 & 1 & 3 & A3-A7 \\
\hline 1 & 8 & $31210 ?$ & 107655 & 6.2 & 1 & 1 & 168 & 5 & 1061 & 2 & 5 & 1 & A1- \\
\hline 1 & 2 & $31320 ?$ & 107966 & 5.2 & 54 & 2 & 174 & 6 & 1111 & 3 & 6 & 2 & $\mathrm{~A} 2-\mathrm{A} 6$ \\
\hline 2 & 9 & $33450^{*}$ & 115708 & 7.8 & 164 & 3 & 194 & 2 & 766 & 6 & 0 & 4 & A3 SrEu \\
\hline 4 & 9 & 33470 & 115735 & 5.2 & -33 & 1 & 145 & 0 & 942 & 0 & 2 & 2 & Hewk Si \\
\hline 1 & 9 & 33550 & 116235 & 5.9 & 52 & 1 & 206 & 2 & 980 & 3 & 16 & 2 & $\mathrm{~A} 2-\mathrm{A} 8$ \\
\hline 1 & 9 & 33560 & 116303 & 6.4 & 160 & 1 & 178 & 2 & 848 & 3 & -3 & 2 & A5-A9 \\
\hline 1 & 9 & 33610 & 116542 & 7.1 & 130 & 2 & 227 & 1 & 866 & 20 & 9 & 1 & A3- \\
\hline 1 & 9 & $33660^{*}$ & 116657 & 4.0 & 60 & 1 & 234 & 1 & 930 & 2 & 10 & 1 & A1-A7 \\
\hline$?$ & 9 & 33850 & 117361 & 6.4 & 254 & 0 & 176 & 2 & 599 & 3 & -6 & 2 & $\mathrm{~F} 1 \mathrm{Sr}$ \\
\hline 2 & 9 & $34020^{*}$ & 118022 & 4.9 & -14 & 6 & 230 & 2 & 942 & 10 & 51 & 0 & $\mathrm{~A} 2 \mathrm{CrEuSr}$ \\
\hline 2 & 9 & $34080 \backslash$ & 118214 & 5.6 & -9 & 3 & 158 & 2 & 1007 & 2 & 3 & 1 & $\mathrm{~A} 0 \mathrm{Si}$ \\
\hline$?$ & 9 & $34270 ?$ & 118623 & 4.8 & 153 & 1 & 175 & 1 & 906 & 5 & 1 & 1 & A 8 \\
\hline 1 & 9 & 34380 & 119082 & 7.4 & 146 & 1 & 232 & 3 & 757 & 7 & 8 & 4 & A3-F0 \\
\hline 2 & 9 & $34410^{*}$ & 119213 & 6.3 & 36 & 3 & 248 & 8 & 864 & 7 & 26 & 2 & A3 $\mathrm{SrCr}$ \\
\hline$?$ & 9 & $34540 ?$ & 119765 & 6.0 & -3 & 3 & 177 & 4 & 1039 & 3 & 1 & 2 & $\mathrm{~A} 1$ \\
\hline 2 & 9 & $34660^{*}$ & 120198 & 5.7 & -52 & 0 & 204 & 0 & 915 & 0 & 38 & 0 & A0 $\mathrm{EuCr}$ \\
\hline 1 & 10 & 49580 & 177983 & 7.3 & 248 & 1 & 240 & 0 & 741 & 3 & 2 & 3 & A5-F2 DD \\
\hline 3 & 10 & 49620 & 178065 & 6.6 & 70 & 2 & 63 & 6 & 726 & 1 & -13 & 4 & B9 HgMn \\
\hline 1 & 10 & 49630 & 178265 & 7.1 & 154 & 1 & 215 & 6 & 764 & 2 & 3 & 4 & F0-F3 \\
\hline 1 & 10 & 49650 & 178327 & 7.9 & 196 & 2 & 221 & 2 & 709 & 8 & -4 & 2 & A6-F5 \\
\hline 1 & 10 & 49690 & 178661 & 7.9 & 146 & 3 & 214 & 3 & 782 & 7 & -2 & 1 & A3-F0 \\
\hline 1 & 10 & 49730 & 178875 & 7.8 & 189 & 0 & 213 & 1 & 851 & 6 & 3 & 5 & A8-F3 \\
\hline 1 & 10 & 49770 & 179143 & 6.8 & 219 & 7 & 254 & 14 & 728 & 15 & 1 & 1 & A4-F5 \\
\hline$?$ & 10 & $49810 ?$ & 179367 & 7.4 & 116 & 1 & 233 & 0 & 858 & 5 & 1 & 1 & A5 \\
\hline 1 & 10 & $49850 ?$ & 179461 & 7.8 & 186 & 5 & 223 & 3 & 730 & 5 & -1 & 2 & A3-F0 DD \\
\hline 2 & 10 & $49860^{*}$ & 179527 & 6.0 & 3 & 1 & 117 & 1 & 793 & 6 & 26 & 0 & B9 SI \\
\hline 2 & 11 & $54660 ?$ & 196133 & 6.7 & 5 & 1 & 179 & 3 & 1021 & 1 & 5 & 0 & A1 SiSr \\
\hline 2 & 11 & $54690^{*}$ & 196178 & 5.8 & -74 & 0 & 142 & 2 & 475 & 3 & 35 & 1 & B8 Si \\
\hline 1 & 11 & 54700 & 196240 & 8.0 & 93 & 3 & 216 & 1 & 1091 & 13 & 7 & 2 & $\mathrm{~A} 4-\mathrm{F} 0$ \\
\hline 1 & 11 & $54730 ?$ & 196362 & 6.4 & 82 & 6 & 197 & 3 & 1093 & 2 & 2 & 1 & $\mathrm{~A} 2-\mathrm{A} 7$ \\
\hline 1 & 11 & 54740 & 196425 & 7.9 & 121 & 5 & 249 & 5 & 979 & 3 & 17 & 3 & A7-F2 \\
\hline 1 & 11 & $54790 ?$ & 196524 & 3.6 & 286 & 1 & 177 & 0 & 540 & 1 & -11 & 2 & $\mathrm{~F} 5 \mathrm{Sr}$ \\
\hline 1 & 11 & $54820 ?$ & 196544 & 5.4 & 18 & 1 & 192 & 3 & 1013 & 8 & 3 & 5 & A2-A5 \\
\hline 2 & 11 & 54840 & 196606 & 6.3 & -44 & 2 & 127 & 2 & 628 & 1 & 20 & 2 & B9 Si \\
\hline 1 & 11 & 54880 & 196655 & 8.0 & 46 & 4 & 186 & 2 & 951 & 1 & 34 & 1 & $\mathrm{~A} 2-$ \\
\hline 3 & 11 & 54920 & 196821 & 6.1 & -13 & 0 & 122 & 1 & 1028 & 1 & 14 & 2 & B9- (HgMn) \\
\hline 2 & 13 & 56860 & 204131 & 6.6 & 0 & 5 & 158 & 5 & 1098 & 1 & 15 & 4 & B9 $\mathrm{SiCrSr}$ \\
\hline 1 & 13 & 56880 & 204188 & 6.1 & 143 & 1 & 201 & 1 & 754 & 1 & 2 & 3 & A5-F0 \\
\hline 1 & 13 & 56890 & 204262 & 7.9 & 116 & 8 & 238 & 4 & 843 & 12 & 4 & 2 & $\mathrm{~A} 0-$ \\
\hline 2 & 13 & $56920 ?$ & 204411 & 5.3 & 48 & 3 & 190 & 2 & 1184 & 1 & 19 & 2 & $\mathrm{~A} 6 \mathrm{Cr}$ \\
\hline 1 & 13 & $56940 ?$ & 204485 & 5.8 & 201 & 2 & 191 & 3 & 645 & 1 & 4 & 4 & F0- \\
\hline 1 & 13 & 56970 & 204541 & 7.5 & 138 & 4 & 232 & 4 & 845 & 1 & 17 & 2 & $\mathrm{~A} 2-\mathrm{F} 1$ \\
\hline 1 & 13 & 57000 & 204751 & 7.9 & 221 & 2 & 224 & 1 & 715 & 8 & 1 & 1 & $\mathrm{~A} 4-\mathrm{F} 6$ \\
\hline 2 & 13 & 57030 & 204815 & 7.2 & 16 & 6 & 139 & 7 & 933 & 4 & 40 & 4 & A0 Si \\
\hline
\end{tabular}


Table 4. c. continued

\begin{tabular}{cclccrrrrrrrrr}
\hline CP & night & RNS & HD & $\operatorname{mag}_{V}$ & $b-y$ & m.e. & $m_{1}$ & m.e. & \multicolumn{1}{c}{$c_{1}$} & m.e. & $\Delta a$ & m.e. & Spt. \\
\hline 2 & 13 & 57070 & 204905 & 7.2 & -4 & 1 & 155 & 4 & 1049 & 0 & 0 & 6 & $\mathrm{~A} 0 \mathrm{Si}$ \\
1 & 13 & 57080 & 204918 & 6.8 & 184 & 1 & 228 & 1 & 764 & 1 & -3 & 2 & $\mathrm{~A} 7-\mathrm{F} 5$ \\
4 & 14 & 58240 & 209339 & 6.7 & 119 & 2 & -15 & 2 & 6 & 4 & -26 & 1 & $\mathrm{Hewk}$ \\
3 & 14 & 58280 & 209459 & 5.8 & -10 & 4 & 119 & 1 & 1054 & 9 & 1 & 1 & $\mathrm{Hg}$ \\
2 & 14 & $58290^{*}$ & 209515 & 5.6 & -17 & 4 & 147 & 1 & 1016 & 4 & 17 & 2 & $\mathrm{CrSiMg}$ \\
1 & 14 & 58360 & 209711 & 8.0 & 156 & 3 & 222 & 2 & 996 & 5 & 8 & 8 & $\mathrm{~A} 3-$ \\
1 & 14 & 58400 & 209845 & 7.2 & 79 & 3 & 225 & 1 & 972 & 6 & 15 & 4 & $\mathrm{~A} 1-\mathrm{F} 1$ \\
1 & 14 & 58430 & 209920 & 7.2 & 147 & 8 & 236 & 1 & 805 & 8 & 9 & 3 & $\mathrm{~A} 2-$ \\
2 & 14 & $58470^{*}$ & 210071 & 6.4 & -42 & 1 & 138 & 2 & 562 & 3 & 18 & 1 & $\mathrm{SiCrHg}$ \\
2 & 14 & 58520 & 210432 & 7.2 & -1 & 2 & 237 & 3 & 895 & 3 & 25 & 2 & $\mathrm{CrSiSr}$ \\
1 & 14 & $58660 ?$ & 211336 & 4.2 & 161 & 0 & 195 & 2 & 764 & 3 & -1 & 1 & $\mathrm{~A} 7-$ \\
1 & 12 & $58700 ?$ & 211433 & 6.9 & 57 & 4 & 215 & 2 & 980 & 15 & 4 & 5 & $\mathrm{~A}-$ \\
1 & 12 & 58750 & 211643 & 7.1 & 48 & 3 & 219 & 2 & 950 & 2 & -3 & 5 & $\mathrm{~A} 3-\mathrm{A} 7$ \\
1 & 12 & 58780 & 211785 & 7.9 & 126 & 3 & 214 & 5 & 854 & 2 & -6 & 4 & $\mathrm{~A} 2-$ \\
1 & 12 & $58790 ?$ & 211797 & 6.2 & 156 & 3 & 210 & 5 & 803 & 1 & 2 & 1 & $\mathrm{~A} 9-$ \\
1 & 12 & 58810 & 211856 & 8.0 & 228 & 5 & 225 & 7 & 695 & 1 & -10 & 9 & $\mathrm{~F} 2-$ \\
1 & 12 & 58840 & 212079 & 8.0 & 139 & 1 & 220 & 5 & 851 & 1 & -2 & 3 & $\mathrm{~A} 0-$ \\
4 & 12 & 58950 & 212454 & 6.2 & -43 & 1 & 102 & 2 & 429 & 4 & 1 & 2 & $\mathrm{Hewk}$ \\
1 & 12 & 58970 & 212595 & 7.4 & 126 & 6 & 234 & 6 & 846 & 3 & 1 & 2 & $\mathrm{~A} 1-\mathrm{A} 9$ \\
1 & 12 & 59080 & 213143 & 7.8 & 222 & 4 & 249 & 4 & 708 & 9 & 2 & 4 & $\mathrm{~A} 5-$ \\
2 & 12 & 59100 & 213232 & 8.0 & 63 & 1 & 220 & 3 & 997 & 7 & 17 & 1 & $\mathrm{~A} 4 \mathrm{Sr}$ \\
\hline
\end{tabular}

Notes to Table 4.:

Col. 1: $\mathrm{CP}$-class

Col. 2: number of night (see Table 2)

Col. 3: Renson number with probability mark:

$$
\begin{array}{ll}
* & \text { well established } \\
\text { blank } & \text { very probable } \\
? & \text { less probable } \\
\backslash & \text { erroneous }
\end{array}
$$

Col. 4: HD number

Col. 5: $\operatorname{mag}_{V}$ from Renson (1991)

Cols. 6-13: own photometry with mean errors (m.e.)

Col. 14: Spectral type according to Renson (1991).

ference in $\Delta a$ is: for HD $71866+9$ mmags, for HD 1157080 mmags and for HD $118022+3$ mmags. Lebedev (1986) has 5 CP2 stars in his catalogue in common with our sample for which the differences in $\Delta a$ are: HD $90044+22$ mmags, HD $118022+10$ mmags, HD $120198+1$ mmags, HD $196178-1$ mmags and HD $204411+5$ mmags. The tendency of slightly larger $\Delta a$ values is expected by the fact that the central wavelength of the $g_{2}$ filter in our system has been slightly shifted to the blue, thereby moving into the deeper part of the 5200 A depression feature.

Comparison with Geneva photometry: in all cases where our $\Delta a$ values indicate photometric non-peculiarity, we also find non-peculiar values of the Geneva indices, i.e. for HD $86274(?), 92728,94334(?), 115708\left(^{*}\right) 118214(\backslash)$, 196133 (?) (the signs in parentheses mark the probability of peculiarity as given by Renson 1991). The photometric evidence obviously supports the assessment of the Rensoncatalogue, taking into account that HD 115708 is a very late type CP2-star (for this type $\Delta a$ only in very rare cases shows peculiar values).

Note on an individual object:

HD 210432 and HD 210433 form a visual binary, for which Renson (1991) because of similar brightness concludes the impossibility to assign peculiarity to one of both. Looking at our uvby and $\Delta a$ values we are in favour of HD 210432 to be the CP2 star and HD 210433 to have spectral type B9.5 V, contrary to Abt (1985) and also to the entry in Renson (1991). HD 210433 has served as normal star in our study for the definition of the normality line.

\section{2. $C P 1$ stars}

Figure 3 shows the diagram $a$ vs. $b-y$ for this group. HD 196655 is outstanding with $\Delta a=34$ mmags. Such a high value has never been observed for a metallic line star. The classification as CP1 star rests on Bidelman (1985) using objective prism spectra. We propose this object for 


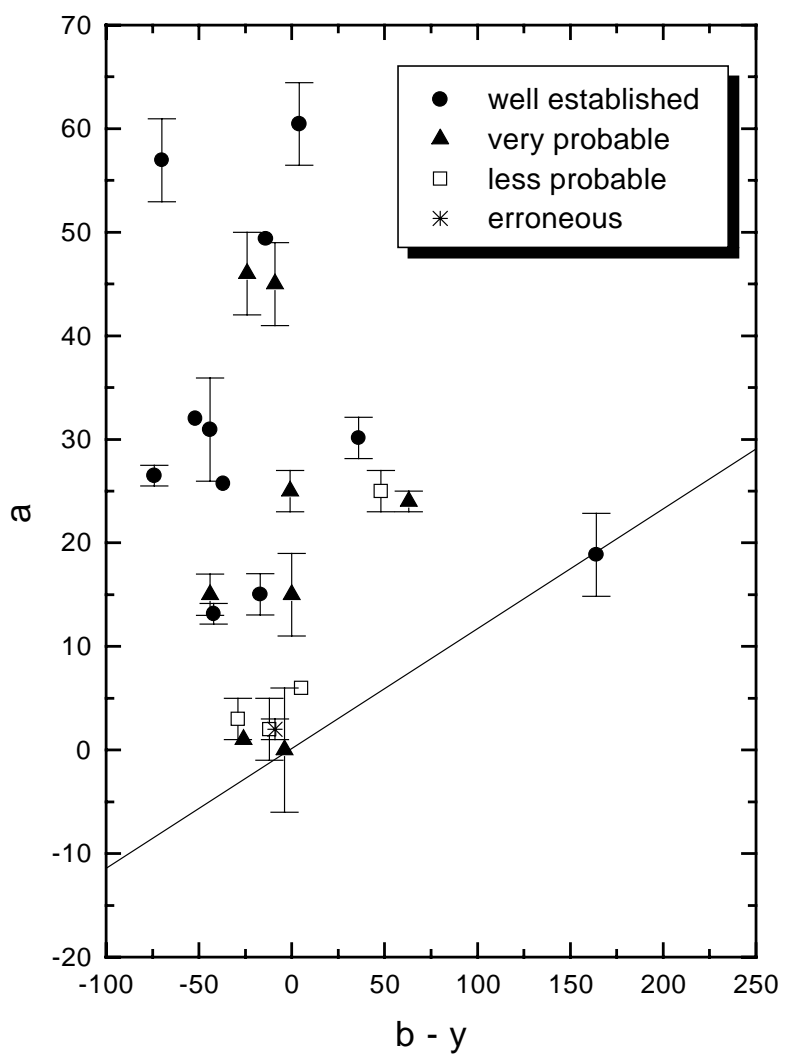

Fig. 2. $a$ vs. $b-y$ for $\mathrm{CP} 2$ stars

reobserving both spectroscopically and photometrically in order to decide whether this star is CP1 or CP2. The same applies also to those five stars which are in such a distance above the normality line that they would be individually considered to be moderate CP2 stars if no other information should be available: HD 107168, 116235, 196425, 204541 and 209845 with $\Delta a=18,16,17,17$ and 15 , resp.

Only one star deviates significantly below the normality line: the $\delta$ Del star HD 79066 with $\Delta a=-16$ mmags.

Discarding the 6 cases of significant deviations above the normality line and those cases with question mark in the Renson-catalogue we arrive at the conclusion that the average deviation of CP1 stars above the normality line is less than one standard deviation from the mean of the group.

A note is due to the following CP1 star:

HD 209711=RNS 58360 has a published value, cited in Renson (1991) $m_{1}=137$ mmags. Considering the other Strömgren parameters this would mean that the star is metal deficient, not Am as listed in the Renson catalogue (A3-). Since this star exhibits the largest difference between observed and published $\mathrm{m}_{1}$ by far, we tend to assume that the correct value would be clearly larger than 200 mmags and that perhaps 137 should be replaced by 237.

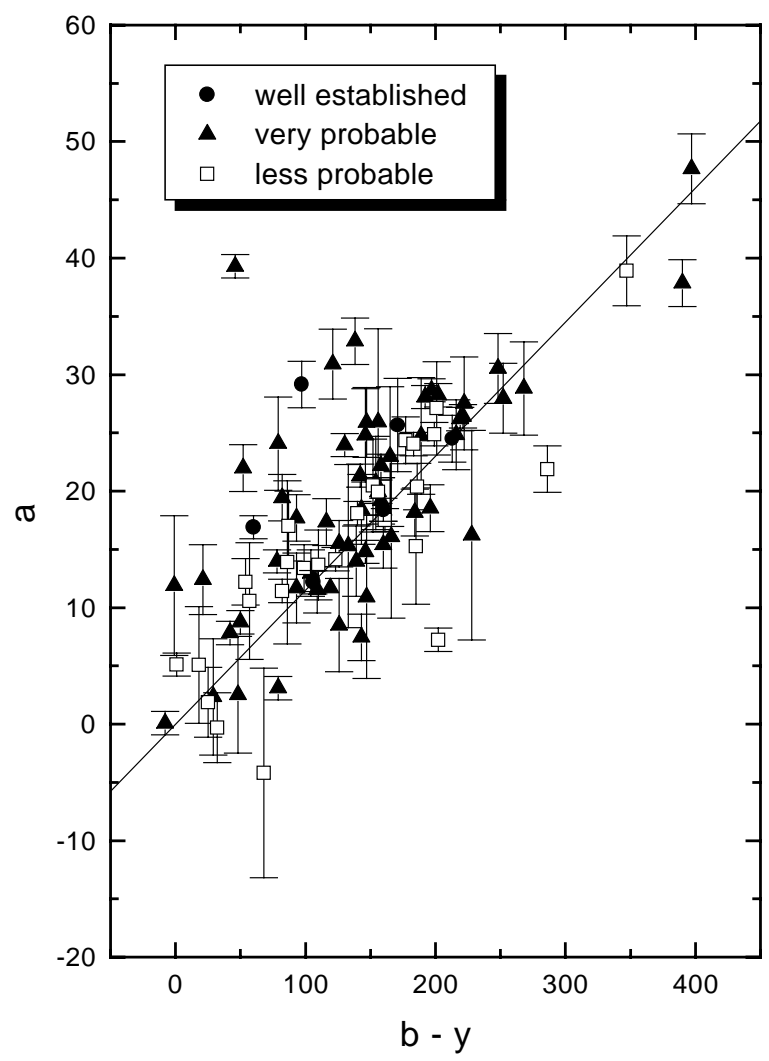

Fig. 3. $a$ vs. $b-y$ for $\mathrm{CP} 1$ stars

\section{3. $C P 3$ stars}

Our 8 stars are very close to the normality line in Fig. 4 with one exception: HD 196821 which has $\Delta a=$ 14 mmags, and thus is just at the threshold of peculiarity in this index. This means that at such low levels of photometric peculiarity one has to be careful to assign the star to the CP2 group as well as in the case of CP1 stars with similar $\Delta a$ values. Spectroscopy has also to be improved in order to establish the final type. The slightly trend towards positive $\Delta a$ values for the CP3 stars of our sample is in good agreement with the results of North (1984).

We should add a note here concerning $\Delta a$ values for three CP3 stars of our sample common to the catalogue of Lebedev (1986): for HD 77350, 78316 and 89822 he derived (from spectrophotometric scans) $\Delta a=18,15,16$, resp. while our $\Delta a$ photometry yields $1,1,6$, resp. It would be interesting to find out the reason for the peculiarity of Lebedev's synthetic $\Delta a$ values.

\subsection{CP4 stars}

Including one He strong star HD 209339 in this grouping we notice that only one of those 5 stars has a peculiar $\Delta a=31$ : HD 79158 (Fig. 4) and therefore may be considered as magnetic peculiar star. 


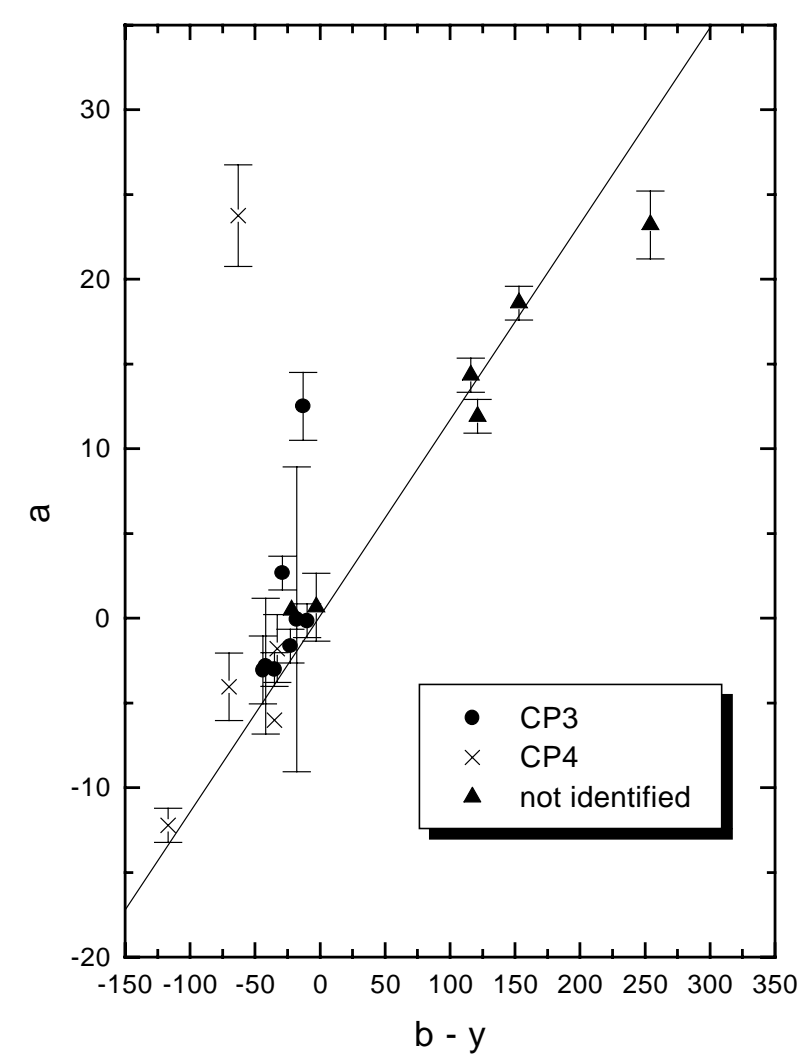

Fig. 4. $a$ vs. $b-y$ for $\mathrm{CP} 3, \mathrm{CP} 4$ and unidentified stars

\subsection{CP? stars}

This group consists of 6 stars and is characterized by the fact that from the spectral type in the Renson catalogue it is not possible to derive any conclusion why those stars have received an entry in the catalogue. Our $\Delta a$ values are neutral concerning this question, since all stars are close to the normality line.

\section{Conclusions}

1. We have provided Strömgren and $\Delta a$ values for a sample of 131 stars in the Renson (1991) catalogue of Ap and Am stars. While 84 percent of the stars have already been measured in the Strömgren system, only 10 percent have previous measurements in $\Delta a$. The average deviations from published Strömgren photometry are comparable to the published accuracy with the exception of very few cases which could be ascribed to intrinsic variability, and in one case (HD 209711) where we are suspicious about a typographical error. $\Delta a$ photometry agrees well with previously published values, but has a slight tendency for enhanced $\Delta a$ due to the position of the $g_{2}$ filter. Only the synthetic $\Delta a$ values of Lebedev (1986) for 3 CP3 stars in common are systematically too large.

2. Renson's (1991) assignment of peculiarity probability is in excellent agreement with our $\Delta a$ results for the magnetic peculiar stars, i.e. CP2. The behaviour of 2 rather probable Ap stars which show non-peculiar $\Delta a$ is an exception to this situation. Further observations may clear up the question, whether variability could be responsible.

3. This study has confirmed with a sufficiently large data base that CP1 stars are photometrically normal in the $\Delta a$ system with the exception of very few stars close to the $3 \sigma$ threshold and one star, HD 196655, which may be a CP2 star.

We have found one CP4 star to be peculiar in $\Delta a$, and one CP3 object just at the threshold.

4. In very good accord with the results of Maitzen \& Vogt (1983) Geneva photometry is helpful with parallel results on photometric peculiarity. Especially we have to outline that there was no case among the CP2 group where photometric peculiarity was found in the Geneva system and not in $\Delta a$.

5. Finally, we have to emphasize that our results have been obtained at the required level of accuracy which was possible only through the use of a non-classical photoelectric technique in an astroclimate (central Europe), which is rather different from excellence.

Acknowledgements. Our thanks go to Dr. M. Stoll who kindly provided software and hardware for the photometer and the telescope control system used in this work. We express special gratitude to Mrs. E. Pressberger for her untiring assistance during the observations. The project was supported by the Hochschuljubiläumsstiftung der Stadt Wien (to HMM, under Wiener Zweikanalphotometer). EP acknowledges support of this research within the working group Asteroseismology$A M S$ with funding from the Fonds zur Förderung der wissenschaftlichen Forschung (project S7303-AST). Use was made of the Simbad database, operated at CDS, Strasbourg, France.

\section{References}

Abt H.A., 1985, ApJS 59, 95

Bidelman W.P., 1985, AJ 90, 341

Lebedev V.S., 1986, Astrofiz. Issled. 21, 30

Maitzen H.M., 1976, A\&A 52, 223

Maitzen H.M., Seggewiss W., 1980, A\&A 83, 328

Maitzen H.M., Vogt N., 1983, A\&A 123, 48

Maury A.C., 1897, Ann. Obs. Harvard 28, part 1

North P., 1984, Ph.D. Thesis, Université de Lausanne

Pressberger R., Stoll M., 1993, Proc. IAU Coll. No. 136, Elliot I. and Butler C.J. (eds.). Dublin Inst. Adv.Stud., p. 86

Preston G.W., 1974, ARA\&A 12, 257

Renson P., 1991, Catalogue Général des Étoiles Ap et Am, Institut d'Astrophysique Université de Liège

Rufener F., 1988, Catalogue of Stars measured in the Geneva Observatory Photometric system (fourth edition), Observatoire de Genève 